\title{
Erratum: Search for Axionlike-Particle-Induced Prompt $\gamma$-Ray Emission from Extragalactic Core-Collapse Supernovae with the Fermi Large Area Telescope [Phys. Rev. Lett. 124, 231101 (2020)]
}

\author{
Manuel Meyer® \\ (Fermi-LAT Collaboration) \\ Tanja Petrushevska \\ (Received 4 August 2020; published 9 September 2020)
}

DOI: 10.1103/PhysRevLett.125.119901

Due to an erroneous unit conversion in the calculation of the expected axionlike-particle (ALP) flux produced during a core collapse supernova (SN), this flux, denoted as $d \dot{N}_{a} / d E$ in Eq. (1) in the main Letter [1] was overestimated by a factor of 100 . Since this flux scales as $g_{a \gamma}^{2}$, all limits reported in the Letter degrade by a factor of $\sim 10$. We have fixed the bug in our numerical calculations and have rerun the analysis. The corrected results, together with the updated plots of the main text of the Letter, are presented in the first section, where we also discuss updates to the conclusion. In the second section, the corrections to the Supplemental Material are presented.

Corrections to the abstract and main text.-In Fig. 1, we show the updated limits for SN2017ein, which are presented in the right panel of Fig. 1 in the Letter. As expected, the limits have degraded by a factor of 10. The same is observed for the combined limits, and the updated Fig. 2 of the Letter is shown in Fig. 2. As a result, the quoted numbers in the Discussion section of the main Letter change in the following way.

With the nonobservation of a $\gamma$-ray burst from a sample of 20 extragalactic core-collapse $\mathrm{SNe}$, we are able to rule out photon-ALP couplings $g_{a \gamma}>\left(2.6_{-0.7}^{+2.0}\right) \times 10^{-11} \mathrm{GeV}^{-1}$ below $m_{a}<3 \times 10^{-10} \mathrm{eV}$ at $95 \%$ confidence under the assumption that all progenitors have a mass of $10 M_{\odot}$, that the optical emission can be modeled with the NiCo model in MOsfiT, and that the GMF is given by the model of JF12. Accordingly, the last sentence of the abstract should therefore read: "Under the assumption that at least one SN was contained within the LAT field of view, we exclude photon-ALP couplings $\gtrsim 2.6 \times$ $10^{-11} \mathrm{GeV}^{-1}$ for ALP masses $m_{a} \lesssim 3 \times 10^{-10} \mathrm{eV}$, within a factor of $\sim 5$ within previous limits from SN1987A".

Furthermore, changing the GMF model, the optical light curve model, or setting the progenitor mass to $18 M_{\odot}$, still has only marginal influence on the constraints and the limits end up between $g_{a \gamma}<(2.1-3.2) \times 10^{-11} \mathrm{GeV}^{-1}$ for $m_{a}<3 \times 10^{-10} \mathrm{eV}$. When increasing the progenitor mass to $40 M_{\odot}$, the limits now improve to $g_{a \gamma}<7.9 \times 10^{-12} \mathrm{GeV}^{-1}$.

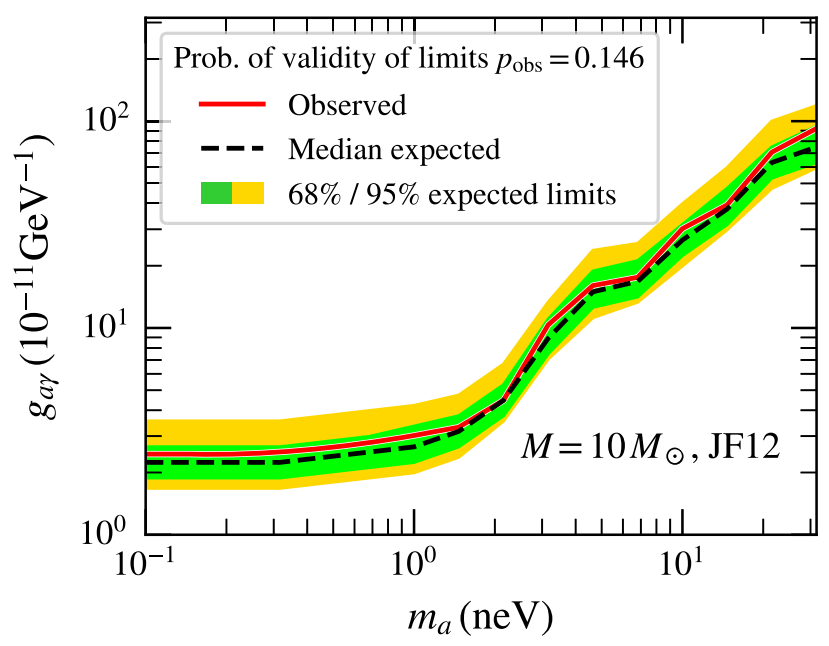

FIG. 1. Updated limits for SN2017ein, replacing the right panel of Fig. 1 in the Letter. 


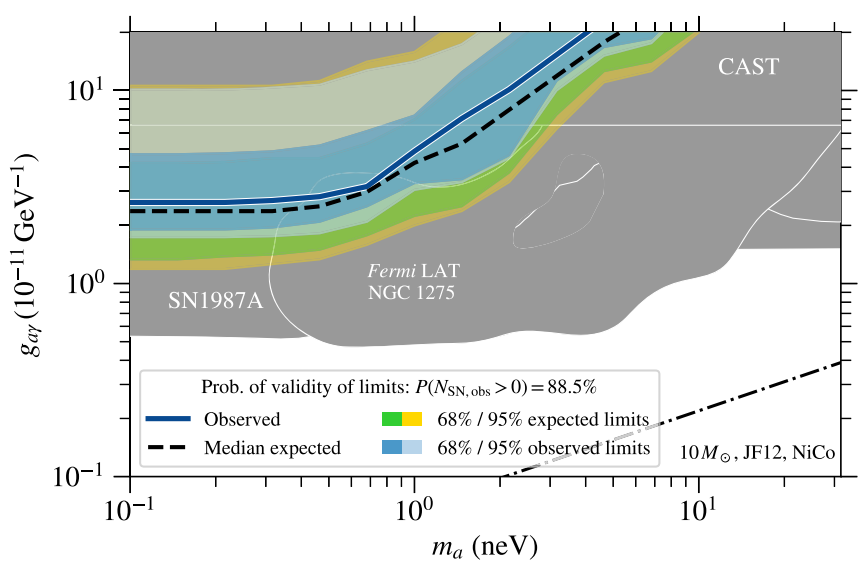

FIG. 2. Updated Fig. 2 of the Letter showing the combined limits in comparison to other exclusions.
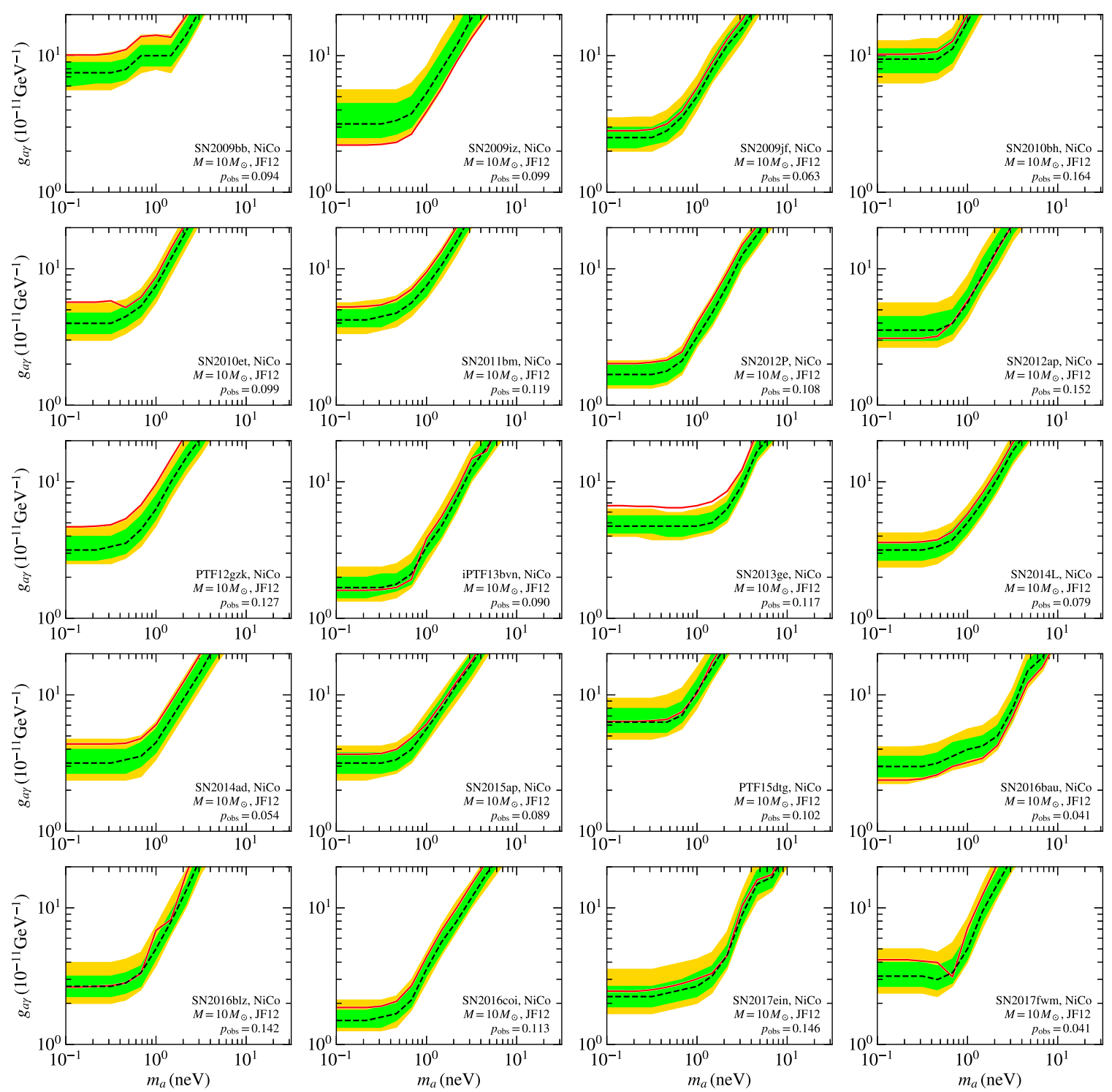

FIG. 3. Updated Fig. 8 of the Supplemental Material, showing the limits for each individual SN. 

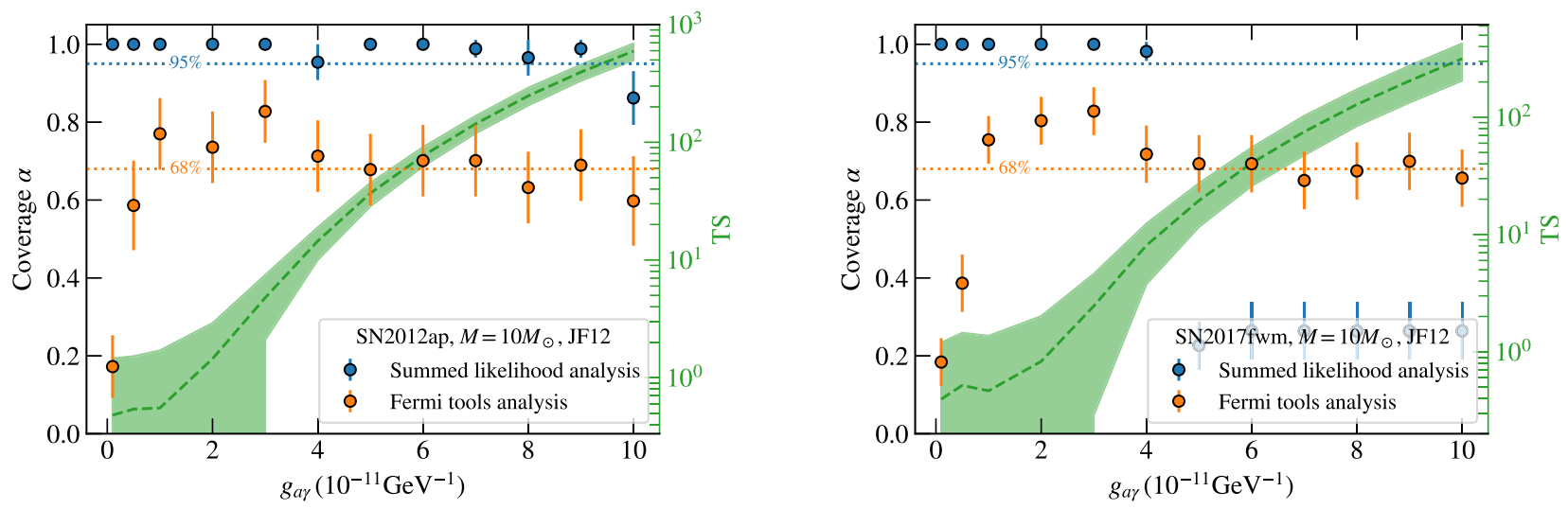

FIG. 4. Updated results for the coverage study; this replaces Fig. 9 of the Supplemental Material.
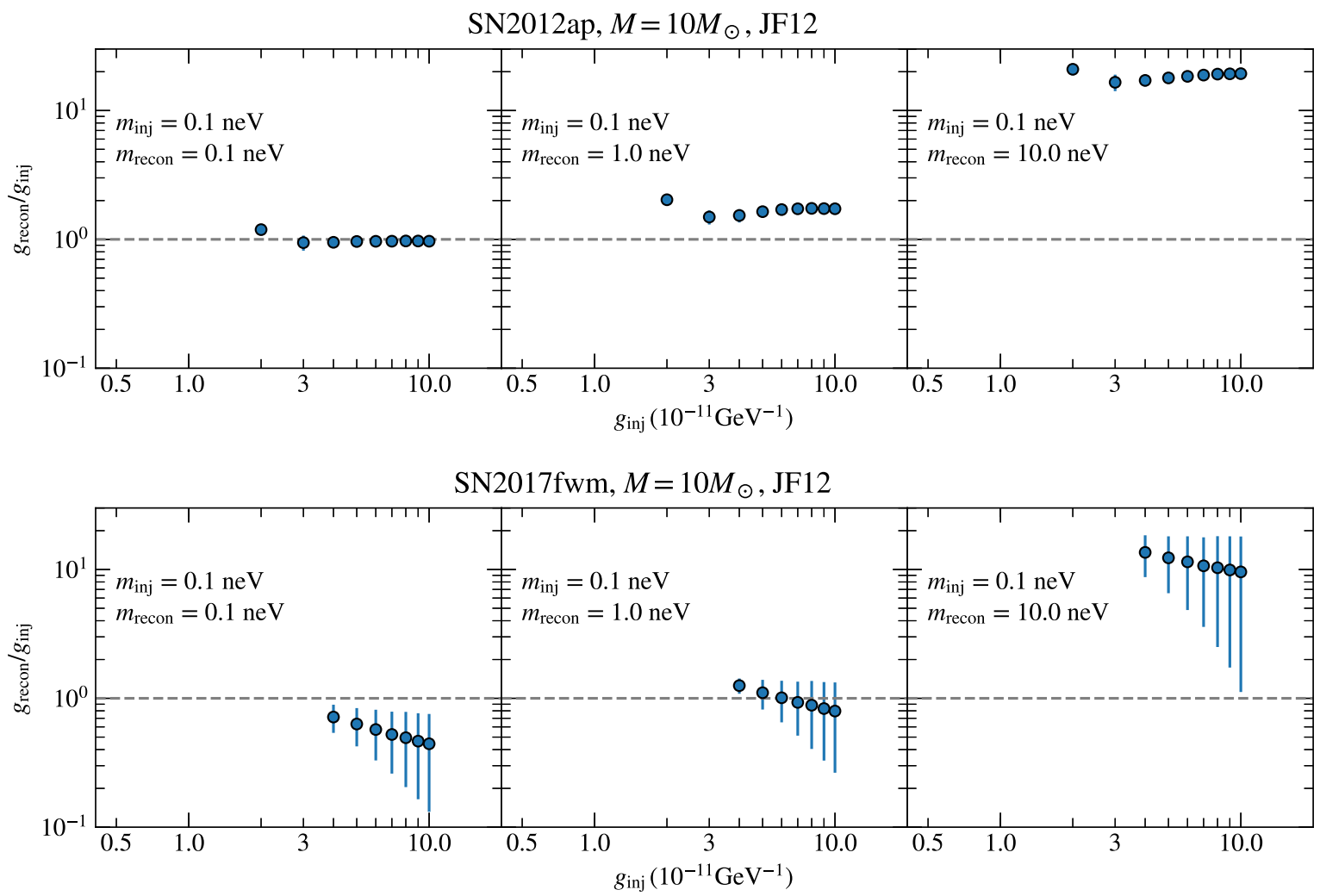

FIG. 5. Updated results for the reconstructed coupling values for simulated signals; this replaces Fig. 10 of the Supplemental Material.

As a consequence of the degraded limits, the third and fourth paragraph in the discussion section of the main Letter should instead read "Our analysis is able to put constraints in the mass range $10^{-3} \mathrm{neV} \lesssim m_{a} \lesssim 1 \mathrm{neV}$ that are within a factor of 5 of the limits obtained from SN1987A for our fiducial set of model parameters (our limits extend to arbitrary low masses since the photon-ALP conversion probability in the Galactic magnetic field is independent of mass for $m_{a} \lesssim 0.5 \mathrm{neV}$ ). These constraints agree with the expectation that the limits scale as $\sqrt{d}$; for a potential SN in the Andromeda Galaxy $(d=778 \mathrm{kpc})$ limits of the order of $g_{a \gamma} \sim 2 \times 10^{-12}$ should be possible [2]."

Corrections to the Supplemental Material.-In Fig. 3, we show the updated band constraints from the individual SNe, which supersedes Fig. 8 of the Supplemental Material. All observed limits now agree very well with the expected constraints.

We have updated the coverage test and now test injected signals for $g_{a \gamma}=(0.5,1,2, \ldots, 10) \times 10^{-11} \mathrm{GeV}^{-1}$. The results of the updated coverage test are shown in Fig. 4 and the reconstructed values of the coupling for simulated injected signals 


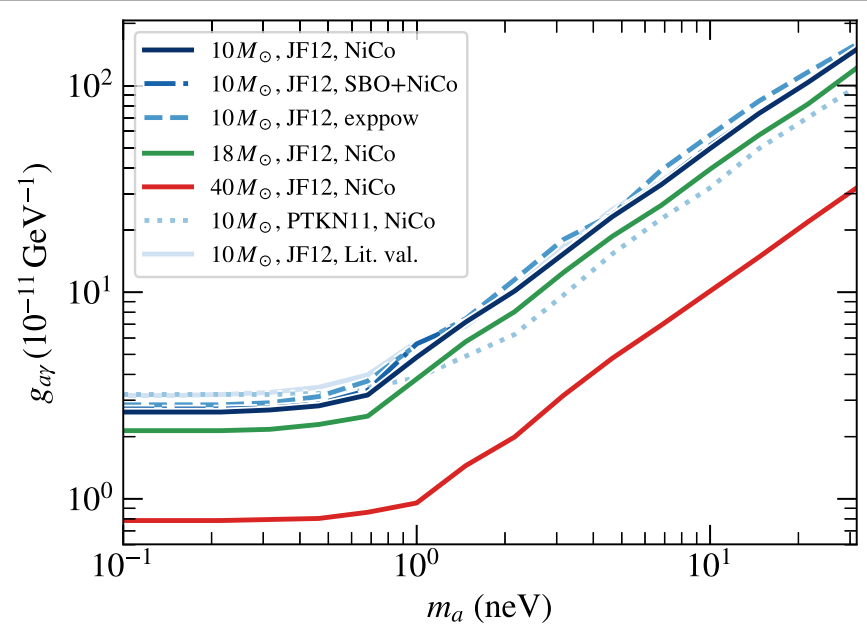

FIG. 6. Updated median limits for different model assumptions to compute the limits on the photon-ALP coupling. This Figure updates Fig. 11 of the Supplemental Material.

are shown in Fig. 5. These replace Figs. 9 and 10 in the Supplemental Material. The injected signal is detected for $g_{a \gamma} \gtrsim 4 \times 10^{-11} \mathrm{GeV}^{-1}$, above which the bin-by-bin analysis for SN2017fwm results in undercoverage.

Lastly, Fig. 6 shows the updated constraints when model assumptions are changed. Again, the limits degrade by a factor of 10 .

[1] M. Meyer and T. Petrushevska (Fermi-LAT Collaboration), Search for Axionlike-Particle-Induced Prompt $\gamma$-Ray Emission from Extragalactic Core-Collapse Supernovae with the Fermi Large Area Telescope, Phys. Rev. Lett. 124, 231101 (2020).

[2] M. Meyer, M. Giannotti, A. Mirizzi, J. Conrad, and M. A. Sánchez-Conde, Fermi Large Area Telescope as a Galactic Supernovae Axionscope, Phys. Rev. Lett. 118, 011103 (2017). 